\title{
Verbes de rupture simples et réfléchis: Deux constructions intransitives
}

\author{
Michel Achard \\ Rice University \\ achard@rice.edu
}

\section{Introduction}

Selon la tradition 'sémantique' de l'analyse des verbes intransitifs de changement d'état (voir par exemple Bailly 1932), la distinction entre les formes simples (rougir), et réfléchies (se rougir) est imputable à la localisation spécifique de la force tenue pour responsable du changement d'état (Rothemberg 1974, Forest 1988, Labelle 1992). La forme simple indique que l'entité qui subit le changement d'état en porte l'exclusive responsabilité : "Lorsque cet élément assume la fonction de sujet de l'intransitif, il est considéré comme possédant, de par lui même, les qualités nécessaires permettant (ou empêchant) la réalisation du processus" (Rothemberg 1974 : 138). Pour Forest (1988), les formes simples manifestent des propriétés entéléchiques, en ce sens qu'elles désignent la réalisation d'un processus interne à une entité: "On peut retenir le terme entéléchique parce qu'il exprime bien, croyons nous, la différence qui oppose La branche casse à La branche se casse, du point de vue du verbe employé. Il ne faudrait pas philosopher à partir du réfléchi de se casse; il n'exprime pas l'auto- affectation, mais précisément le changement d'état purement extérieur aux virtualités de l'entité considérée. Par contre, une branche ne casse pas si cela ne correspond pas à une altérabilité de la branche; et quand on dit La branche casse, on prend en compte dans la description la conformité du changement à une virtualité intime » (Forest 1988: 152).

Ce type d'analyse a indéniablement porté ses fruits. Par exemple Labelle (1992: 391) montre avec bonheur que la distinction entre Jeanne rougit/*se rougit et il vit le mouchoir *rougir/se rougir soudain est motivée par le fait qu'un être humain possède en elle même toutes les qualités nécessaires pour amener le rougissement à bien, tandis qu'un mouchoir, en étant incapable, doit se reposer sur l'apport d'éléments extérieurs. Une analyse similaire pourrait également rendre compte de la différence entre les paires pourrir/*se pourrir, ou refroidir/se refroidir par exemple.

Il ne semble pas, cependant, que l'hypothèse entéléchique soit en mesure de rendre compte de l'ensemble des verbes de changement d'état. En particulier, et cela malgré l'exemple originel de Forest, elle rencontre de réelles difficultés avec les verbes de rupture (casser, briser, rompre). En contexte, il semble en effet difficile d'imputer le choix du réfléchi ou de la forme simple en (1) au côté interne ou externe de la force responsable du bris, puisque dans les deux cas, la configuration dynamique qui le provoque (un homme qui s'accroche à l'entité qui rompt) est identique:

(1) a. Le jeune homme est monté sur le rebord d'une fenêtre au troisième étage de l'établissement et a voulu descendre en s'accrochant à la branche d'un arbre, mais elle a cassé, indique le journal The Statesman (AFP)

b. - il y a un homme à l'eau! Dis- je, le coeur serré. - il s'est accroché au bout du mât, qui s'est cassé, poursuivit Barnavaux. (Mille P. Barnavaux et Quelques Femmes)

Afin de rendre compte des données en (1), ainsi que d'autres aspects pertinents aux verbes de rupture, cette présentation explore les verbes exploser, éclater, ainsi que les précités briser, casser et rompre dans un corpus journalistique de 70 millions de mots, ainsi que dans 514 textes du $20^{\text {ème }}$ siècle du projet FRANTEXT ARTFL. L'hypothèse proposée est que la présence de la forme simple ou réfléchie est imputable non pas au siège de la force responsable pour la rupture, mais au niveau de participation du sujet de l'intransitif dans la configuration dynamique décrite par le verbe. Les formes simples $(\varnothing)$ et 
réfléchies (se) représentent deux constructions spécifiques dans le sens de Goldberg (1995) et Langacker (1987, 1991), qui codent deux configurations dynamiques différentes (Talmy 1988). Se décrit l'interaction entre deux sources d'énergie (parmi elles le sujet de l'intransitif), tandis que $\varnothing$ n'en reconnait qu'une. De ce fait, se met en valeur la participation active du sujet de l'intransitif dans le processus verbal, tandis que $\varnothing$ l'occulte. Le sens respectif des constructions se et $\varnothing$ se combine avec les traits lexicaux spécifiques des verbes, ainsi que les propriétés plus générales des conditions du discours, pour rendre compte de la distribution attestée dans le corpus.

Cette présentation est organisée de la façon suivante: La section 2 présente brièvement l'origine de l'hypothèse proposée. La section 3 introduit la distribution des deux constructions avec les 5 verbes. La section 4 récapitule les résultats et considère l'apport possible de l'hypothèse entretenue ici pour un traitement global des verbes de changement d'état.

\section{Les réfléchis 'énergiques'}

Maldonado (1988) décrit pour l'espagnol une alternation beaucoup plus générale entre $\varnothing$ and se, comme le montrent les exemples (2) et (3) (tirés de Maldonado 1988):

(2) La pelota cayó (*se) de la mesa como era esperado 'La balle tomba de la table comme prévu'

(3) La pelota se cayó (Ø) de la mesa inesperadamente 'La balle tomba de la table de façon inattendue'

Dans l'exemple (2), où la chute de la balle est prévisible, $\varnothing$ représente le seul choix possible. En (3), où la chute est inattendue, se est choisi de façon exclusive. Maldonado explique la présence systématique de se avec des processus inattendus par le fait que la construction réfléchie est plus 'énergique' que son alternative: "I will propose that the reflexive construction is used when there is a situation of opposing forces in which one force overcomes the other. Generally speaking, natural expectations and canonical conceptions of world events constitute the resisting force being overcome" "Je propose que la construction réfléchie est utilisée dans une situation de forces opposées dans laquelle l'une des forces domine l'autre. En termes généraux, le déroulement attendu des évènements dicté par notre conception du monde constitue la force dominée' (Maldonado 1988: 155, ma traduction). Il va de soi que l'analyse de Maldonado pour l'espagnol ne peut pas être directement adoptée ici parce que le français n'est pas aussi systématique que l'espagnol dans sa sélection des deux constructions, comme le montre l'exemple en (4) où le réfléchi est également impossible que la chute soit prévue ou pas:

\section{(4) La balle (*s') est tombée de la table comme on le pensait/de façon inattendue}

Cependant, même si le français ne fait pas usage du côté énergique des réfléchis d'une manière aussi systématique que l'espagnol, cet aspect n'en figure pas moins de façon importante dans l'analyse de la distribution de se et $\varnothing$ parce que ces deux constructions se distinguent en premier lieu d'un point de vue sémantique par les différentes configurations dynamiques qu'elles imposent sur la scène qu'elles décrivent.

Toutes les situations de rupture ont en commun une interaction énergique lors de laquelle une entité subit un changement d'état radical. Les deux constructions se et $\varnothing$ représentent deux façons différentes de structurer cette interaction, ou en d'autres termes, deux configurations dynamiques différentes. Se met en valeur la participation du sujet de l'intransitif à l'interaction dynamique que le verbe décrit. Cette participation prend la forme de la résistance qu'elle oppose à la force supérieure qui lui impose son changement d'état. La construction en se met en valeur l'aspect dynamique de l'interaction en présentant l'entité qui casse comme capable de résistance. Deux entités énergiques sont présentées face à face avant que la force dominatrice ait raison de la résistance du sujet de l'intransitif. Par contraste, la construction $\varnothing$ code le manque de résistance du sujet de l'intransitif pendant l'interaction dynamique. Elle passe sous silence le côté dynamique de l'échange en mettant en avant le manque de résistance du sujet de l'intransitif. La suite de cette présentation montre que la structure lexicale des verbes briser et exploser et 
éclater les rend respectivement incompatibles avec les constructions $\varnothing$ et se, alors que certains traits sémantiques ainsi que certaines caractéristiques du contexte se combinent pour expliquer la distribution des deux constructions avec les verbes casser et rompre. ${ }^{1}$

\section{La distribution des verbes de rupture}

Les exemples (5)-(8) illustrent la distribution des constructions $\emptyset$ et $s e$ avec les verbes analysés dans cette présentation. (5) montre qu'exploser et éclater sont uniquement possibles avec Ø. (6) illustre l'exclusivité de briser avec se, tandis que (7) et (8) indiquent que casser et rompre sont compatibles avec les deux constructions:

(5) a. Par ailleurs, à Buenos Aires, une grenade lacrymogène a explosé/*s'est explosé devant l'ambassade du Japon. (AFP)

b. Les troubles qui ont éclaté/"se sont éclaté à la frontière entre l'Azerbaidjan soviétique et l'Iran au début de janvier ont tout autant surpris les autorités de Téhéran que le reste du monde. (AFP)

(6) il me semble qu'une digue se brise/*brise en moi (M. Barrès. Mes cahiers T3)

(7) a. des branches cassèrent, en arrière et de côté (R. Bazin. Le blé qui lève)

b. je m'assieds enfin et la chaise se casse! (J.K. Huysmans. L'oblat)

(8) a. Si l'une des digues venait à rompre, les eaux pourraient submerger des routes secondaires...(AFP)

b. Appel à évacuation immédiat pour un village dont la digue est prête à se rompre (AFP)

Le total des exemples attestés pour les 5 verbes est donné dans le tableau 1, la distribution en pourcentage de Ø et se avec briser, casser, et rompre est donnée dans le tableau 2:

Tableau 1

\begin{tabular}{|c||ccc||ccc||ccc|}
\hline \multicolumn{1}{|c||}{} & \multicolumn{3}{c||}{ Corpus AFP } & \multicolumn{3}{c||}{ Corpus ARTFL } & \multicolumn{4}{c|}{ Total } \\
\cline { 2 - 9 } & $\varnothing$ & se & Total & $\varnothing$ & se & Total & $\varnothing$ & se & Total \\
\cline { 2 - 10 } Exploser & 48 & $\varnothing$ & 48 & 108 & $\varnothing$ & 108 & 156 & $\varnothing$ & 156 \\
Eclater & 87 & $\varnothing$ & 87 & 199 & $\varnothing$ & 199 & 286 & $\varnothing$ & 286 \\
Briser & $\varnothing$ & 80 & 80 & 5 & 345 & 350 & 5 & 425 & 430 \\
Casser & 50 & 29 & 79 & 65 & 91 & 156 & 115 & 120 & 235 \\
Rompre & 29 & 62 & 91 & 28 & 175 & 203 & 57 & 237 & 294 \\
\hline
\end{tabular}

. Nombre total d'exemples avec exploser, éclater, briser, casser, and rompre

Tableau 2 


\begin{tabular}{|l||cc||cc||cc|}
\hline \multirow{2}{*}{} & \multicolumn{2}{|c||}{ Corpus AFP } & \multicolumn{2}{c||}{ Corpus ARTFL } & \multicolumn{2}{c|}{ Moyenne } \\
& $\varnothing$ & se & $\varnothing$ & se & $\varnothing$ & $s e$ \\
\cline { 2 - 7 } Briser & $0 \%$ & $100 \%$ & $1.4 \%$ & $98.6 \%$ & $1.10 \%$ & $98.9 \%$ \\
Casser & $63.3 \%$ & $36.7 \%$ & $41.7 \%$ & $58.3 \%$ & $48.9 \%$ & $51.1 \%$ \\
Rompre & $31.9 \%$ & $68.1 \%$ & $13.8 \%$ & $86.2 \%$ & $19.4 \%$ & $80.6 \%$ \\
\hline
\end{tabular}

Pourcentage de constructions $\varnothing$ et se avec briser, ${ }^{2}$ casser, et rompre

\subsection{Exploser et éclater}

Le sujet d'exploser perd son intégrité structurale brutalement, victime d'une énergie qui trouve son origine à l'intérieur de lui avant de se propager vers l'extérieur. D'un point de vue sémantique, les deux corpus font preuve d'une remarquable homogénéité. Par exemple, les sujets de 21 exemples sur les 48 du corpus AFP sont spécifiquement conçus pour éclater (bombe, Cocktail Molotov, grenade, etc.). 12 exemples décrivent des machines volantes sophistiquées qui présentent de hauts risques d'explosion vu la complexité et la volatilité de leurs moteurs. Enfin, 12 exemples décrivent métaphoriquement un domaine (souvent économique) qui jouit d'un succès instantané. Dans tous ces trois groupes d'exemples respectivement illustrés en (9)-(11), la force responsable pour le changement d'état s'accumule exclusivement à l'intérieur de l'entité en position de sujet de l'intransitif.

(9) Une grenade a été lancée dans la chambre où dormaient une femme et ses trois enfants. Elle a explosé près du lit de Sakin, quatre ans (AFP)

(10) Une fusée américaine Titan 34 D avait par ailleurs explosé au décollage le 18 avril 1986 (AFP)

(11) Enfin, l'industrie de la céramique a littéralement explosé; les traditionnels arts de la table sont devenus très minoritaires (AFP)

Dans un de ses sens illustré en (12) et (13), éclater ressemble à exploser en ce qu'il code une force (souvent métaphorique) qui trouve son origine à l'intérieur du sujet de l'intransitif et se dirige vers l'extérieur. Dans le corpus AFP, ce sens représente 15,9\% des exemples (14 sur 88):

(12) L'histoire a montré que quand un empire éclate, il sème la zizanie entre les nationalités (AFP)

(13) La gérante nous laisse, enfin. Nous éclatons en paroles, en questions, on ouvre les valises. (G. Colette.Claudine A L'Ecole)

Dans la grande majorité des cas cependant, (70 exemples soit $79.6 \%$ dans le corpus AFP), le sujet d'éclater doit son existence à un échange dynamique dont l'énergie crée une nouvelle entité jusque là inconnue. Ce sens du verbe est illustré en (14)-(17):

(14) Ainsi, lorsque, en 1863, éclata le conflit entre la représentation prussienne et le ministère prussien...(J. Jaurès. Etudes Socialistes)

(15) ... après le dîner, le soir, l'orage enfin éclata. (O. Mirbeau. Journal d'Une Femme de Chambre)

(16) Une fusillade éclate, obligeant le général à faire demi-tour (AFP)

(17) Lorsqu'éclate la deuxième guerre mondiale, Alfred Coste-Floret est chargé de cours à la faculté de droit de Strasbourg (AFP) 
La comparaison des situations présentées dans les exemples (12) et (17) illustre parfaitement les configurations dynamiques spécifiques respectivement évoquées par exploser et ce sens d'éclater. L'empire en (12) représente une entité politique stable qui existe de façon continue pendant une certaine période de l'histoire. En ce sens, son existence est indépendante de la dynamique des forces qui mène à sa destruction. Par contraste, la deuxième guerre mondiale en (17) n'existait pas avant qu'une déclaration formelle ne soit prononcée en 1939. En d'autres termes, l'entité qu'on appelle 'guerre' représente le fruit des tensions politiques qui gouvernaient l'Europe à cette époque, et de ce fait, ne doit son existence qu'au jeu de forces qui la crée.

Les exemples en (9)-(17) montrent qu'exploser et éclater présentent deux configurations dynamiques différentes. Avec exploser et le sens d'éclater en (12) et (13), la force destructrice trouve son origine dans l'entité en position de sujet de l'intransitif. Dans le second sens d'éclater en (14)-(17), un jeu de forces indéfinies donne naissance à l'entité décrite par le sujet de l'intransitif. L'incompatibilité de ces deux verbes avec la construction réfléchie se est claire. Aucune des deux configurations dynamiques qu'ils décrivent ne présente le sujet de l'intransitif comme une entité indépendante dont l'intégrité structurale propre lui permet d'offrir une certaine résistance à la force dominatrice qui amène sa destruction. La construction $\varnothing$ représente de ce fait le seul choix possible.

\subsection{Briser}

Dans son sens intransitif, briser code une configuration dynamique dans laquelle le sujet représente une entité structuralement indépendante dans ses conditions normales d'utilisation. Le changement d'état résulte de son contact avec une autre entité (généralement statique) qui lui oppose une force supérieure. Cette configuration met donc en jeu deux forces différentes, ce qui rend briser naturellement compatible avec la construction réfléchie qui met en valeur l'aspect dynamique du sujet de l'intransitif.

Cette configuration dynamique de deux forces en opposition constitue un trait sémantique de briser, et se manifeste de ce fait dans toutes les constructions dans lesquelles le verbe participe. La comparaison de briser et rompre dans la construction transitive avec un objet abstrait (où casser n'apparait que très rarement) est particulièrement édifiante, parce que cette construction décrit l'interaction entre un agent et un patient, et que, de ce fait, la nature spécifique de ces participants (en particulier celle du patient) nous fournit des indications précieuses sur les configurations dynamiques que les deux verbes évoquent. Parmi les 446 exemples de briser et les 570 exemples de rompre réunis dans les deux corpus, un bon nombre de noms se trouvent exclusivement en position d'objet direct de l'un ou l'autre des deux verbes, ainsi que l'illustre le tableau 3. La lecture de ce tableau révèle que briser code l'application d'une force suffisante pour surpasser une entité elle même énergique mais jugée oppressive (révolte, insurrection, blocus, etc.), tandis que rompre décrit en général la rupture inopinée d'un équilibre positif qui mériterait d'être préservé (pacte, marché, relation, etc.).

Tableau 3 
Briser

Force à surmonter

$\leftarrow$
Ramnuo

Equilibre à préserver

Noms qui apparaissent exclusivement en position d'objet direct de briser ou rompre

Cette différence dans les configurations dynamiques respectivement évoquées par briser et rompre se manifeste également à l'examen de la fréquence des noms qui peuvent figurer en position d'objet direct avec les deux verbes (tableau 4). Le tableau 4 montre que les entités les plus fréquentes avec rompre décrivent des états précaires, dont les diverses composantes doivent œuvrer ensemble pour maintenir un équilibre bénéfique (silence, contact équilibre, relation, lien). En revanche les entités les plus fréquentes avec briser constituent une enfreinte à la liberté et doivent de ce fait être détruites (liens, chaines, idoles), ou représentent une présence agressive et menaçante qu'il convient de museler (résistance) :

Tableau 4.

\begin{tabular}{|c|c|}
\hline Briser & Rompre \\
\hline Liens (30) & Silence (135) \\
\hline Cadre (18) & Lien (42) \\
\hline Idoles (16) & Charme (36) \\
\hline Unité (15) ${ }^{3}$ & Cercle (27) \\
\hline Obstacle (13) & Equilibre (22) \\
\hline Chaînes (13) & Relations (15) \\
\hline Résistance (12) & Contact (15) \\
\hline
\end{tabular}

Fréquence des noms en position d'objet direct avec briser et rompre

Cette configuration dynamique de deux forces en opposition particulière à briser se manifeste également dans la construction intransitive, puisque l'entité responsable de la destruction est explicitement exprimée, souvent par une locution prépositionnelle comme en (18) et (19), dans pratiquement la moitié des cas (45.5 AFP; 59.4 ARTFL). En revanche la même entité n'est mentionnée de façon explicite que dans environ 7\% des cas avec rompre (AFP 6.59 ; ARTFL 7.38).

(18) Mais le navire, qui a pris de l'eau par plusieurs voies, s'est brisé en deux contre des rochers, lundi en milieu de journée (AFP) 
La présence de la construction réfléchie avec briser s'explique par le fait que la configuration dynamique évoquée par le verbe met en présence deux forces séparées. Ce trait sémantique rend donc le verbe exclusivement compatible avec la construction en se qui met en valeur l'aspect dynamique du sujet intransitif.

\subsection{Casser}

La structure sémantique de casser rend le verbe compatible avec les deux constructions. Le choix entre se et $\varnothing$ est donc régi par la nature même de l'entité qui subit le changement d'état, les conditions du discours, ainsi que, plus généralement, les conventions linguistiques.

Les objets physiques possèdent généralement l'intégrité structurale nécessaire pour résister aux pressions diverses auxquelles leur fonction les expose. La plus neutre des situations de rupture code généralement cette résistance avec la construction réfléchie, comme dans (20) et (21):

(20) (il prend un vase sur la cheminée et en donne un énorme coup sur la table.) ah! Vous voulez me faire taire? (il donne encore un énorme coup, le vase ne se casse pas.) (Achard M. Jean de la Lune)

(21) D'un grand coup, la porte s'ouvre et bat contre le mur. Toutes les femmes se retournent: Jaume est sur le seuil. Silence. On entend une tasse qui roule de la table, tombe et se casse (Giono J. Colline)

Le choix de la construction simple indique le choix de l'auteur d'occulter la résistance de l'entité en position de sujet de l'intransitif dans l'épisode dynamique qui résulte en son changement d'état. Ce choix est souvent dicté par la reconnaissance que cette entité n'est pas capable de fournir la résistance nécessaire. La construction $\emptyset$ est donc fréquemment choisie, quand le sujet de l'intransitif est vieux, usé, endommagé, ou poussé au delà de ses limites structurales par une utilisation extrême, comme le montrent les exemples en (21)-(23):

(21) car les vieux instruments trop fatigués se raidissent aussi et cassent tout à coup (Pesquidoux J. de. Le Livre de Raison T. 1)

(22) Il s'amusait à manier un couteau dont il faisait plier la lame. Cette lame soudainement cassa (Pourrat H. Les Vaill. A la Belle Berg)

(23) Trop chargées, les branches cassent (Faure E. L'Esprit des Formes)

La participation du sujet de l'intransitif à l'échange dynamique qui produit sa rupture peut aussi être défocalisée quand cet échange ne sert pas directement le but discursif de l'auteur. Par exemple, dans les exemples en (24)-(26), l'accent est mis sur les conséquences de la rupture, plutôt que sur les conditions dynamiques qui l'ont causée:

(24) Dans le chavirage, poursuit-il, le roof (le toit de la cabine) a été complètement arraché, le système de barre a éclaté, les mèches de safran ont cassé ce qui a occasionné une voie d'eau dans le compartiment arrière du bateau (AFP)

(25) il ne peut plus utiliser sa grand-voile au vent portant. Un handicap considérable jusqu'au cap Horn, qu'il partage avec Patrice Carpentier (Nouvel-OBS), dont la bôme a cassé pour la deuxième fois, à l'endroit où elle avait été réparée $(A F P)$ 
(26) Partis depuis décembre, ils accumulent les catastrophes. Dernière en date, une tempête les a surpris entre la République dominicaine et Porto Rico au cours de la traversée du Canal de la Mona. L'arbre de l'hélice a cassé et les navigateurs ont du demander de l'aide par radio (AFP)

Dans les exemples en (24)-(26), les objets qui cassent font tous partie d'une entité plus grande (un bateau). Le bris en lui même n'est pertinent qu'en ce qu'il empêche le bateau de fonctionner convenablement. D'un point de vue narratif, les jeux de forces qui occasionnent la rupture des mèches de safran, de la bôme, ou de l'arbre de l'hélice sont donc secondaires par rapport aux conséquences de ces ruptures pour la bonne marche du bateau. Le choix de la construction Ø représente le choix de l'auteur de passer sous silence l'aspect dynamique du sujet de l'intransitif pour garder l'accent sur les conséquences de la rupture.

Une stratégie narrative similaire régit le choix de la construction simple quand l'entité qui subit la rupture fait partie des informations d'arrière plan, comme en (27):

(27) Ça pouvait être dans les onze heures du matin quand Panturle s'est arrêté pour raccommoder la longe qui venait de casser. Et, juste au moment où il levait la tête, ayant fini, à travers le soleil, maintenant chaud, il a vu un homme debout sur le champ de Marius Aubergier (Giono J. Regain)

Dans l'exemple (27), l'incident de la longe fait partie des circonstances qui mènent à l'observation de la présence d'un homme dans le champ d'Aubergier. La fonction narrative de la rupture consiste uniquement à amener Panturle à lever les yeux. Son aspect 'technique', qui inclut la résistance de la longe, joue un rôle si mineur relatif à cette fonction qu'il peut facilement être passé sous silence. Lorsque la rupture du sujet de l'intransitif est présentée comme une facette prévisible du monde 'tel qu'il est', la configuration dynamique qui l'occasionne est aussi généralement occultée, comme le montre les exemples en (28) et (29):

(28) l'angoisse de la chute dans le vide ...de la corde qui peut casser... Mais les cordes d' aujourd'hui ne cassent plus, avait affirmé Jos-Mari (Peyre J. Matterhorn)

(29) A l'achat, les artichauts, verts ou violacés suivant les variétés, doivent être choisis sans taches brunes au bout des feuilles, signe qu'il y a trop longtemps qu'ils ont été cueillis. Un test facile pour vérifier leur état de fraîcheur: lorsque vous prenez une feuille entre le pouce et l'index, elle doit casser avec un petit bruit sec sans se plier (AFP)

Il reste néanmoins important de signaler que les conditions qui favorisent la défocalisation du rôle du sujet de l'intransitif dans la configuration dynamique évoquée par le verbe ne représentent que des tendances statistiques. Elles s'effacent en particulier devant des priorités narratives plus spécifiques. Une illustration nous en est fournie par l'exemple en (30), où l'objet qui rompt (la ligne d'installation) fait partie d'une entité plus grande (un pacemaker), de façon similaire aux exemples en (24)-(26). Néanmoins, et cela constitue une différence importante avec ces exemples, la ligne d'installation est fortement topicale parce qu'elle représente la seule raison de l'échec répété du pacemaker. La topicalité de la ligne d'installation justifie l'intérêt porté à chaque facette de cette petite pièce, y compris la mention spécifique de sa participation dans la configuration dynamique évoquée par le verbe, et de ce fait l'emploi de la construction réfléchie.

(30) Telectronics et la Food and Drug Administration (FDA) ont tenté de rassurer les 20,000 porteurs de cet équipement en expliquant que le fil d'implantation en forme de crochet ("Encor-J" modèles 330-854 et 033-856), ne sert qu'à implanter l'électrode au montage du pacemaker. Il est inutile ensuite, mais s'est cassé dans le thorax de cinq personnes qui se sont bien remises (AFP)

De façon plus générale, la présence dans le contexte de tout élément mettant en valeur l'aspect dynamique de l'échange favorise le choix de se. Dans les exemples en (31) et (32), conformément à la tendance observée précédemment avec briser, la mention spécifique de la cause de la rupture, c'est à dire l'entité 
statique dont la résistance cause la destruction du sujet intransitif, favorise la présence de la construction en $s e$ :

(31) Le moujik s'approcha aussitôt de la boîte, qui avait été projetée contre le mur du palais;et au moment où il la prit, je vis qu'elle s'était cassée, et quelle contenait le cadavre d'un petit enfant (Larbaud V. Barnabooth)

\section{(32) L'avion a dérapé et poursuivi sa course jusqu'au fleuve où il s'est cassé en trois morceaux (AFP)}

La distribution de se et $\varnothing$ est également influencée par la présence de plusieurs expressions idiomatiques fréquemment utilisées qui contiennent l'une ou l'autre des constructions, comme en (33) qui illustre ça passe ou ça casse attesté 9 fois dans le corpus AFP, et en (34) qui s'inspire de la formule tant va la cruche à l'eau qu'à la fin elle se casse. Dans ces deux exemples, le choix d'une expression idiomatique spécifique comme cadre linguistique ou conceptuel justifie la présence exclusive de la construction présente dans l'idiome.

(33) "Ou ça passe ou ça casse", ironise un soldat de l'escorte en entrant en secteur serbe (AFP)

(34) Le Premier ministre Alain Juppé a fait les frais de la plupart des slogans et des banderoles. Juppé démission, Juppé privilégié, Juppé, il faut nous augmenter pouvait-on entendre dans le cortège tandis qu'une pancarte proclamait Tant va le fonctionnaire à l'austérité, qu'à la fin il se casse $(A F P)$

Enfin, plusieurs aspects de la distribution de se et $\varnothing$ sont fortement conventionalisés. Le premier est illustré en (35). Dans le sens métaphorique de 'voir ses progrès brutalement stoppés' fréquemment attesté dans la littérature sportive, la construction $\varnothing$ représente le seul choix possible.

(35) Flushing Meadow - 7è journée : Sampras passe, Pioline casse (AFP)

D'autre part, les entités abstraites sont quasi exclusivement attestés avec la construction réfléchie (Lagane 1967). Cette particularité permet de désambiguer certaines situations, comme dans les exemples (36) et (37), tous deux prononcés par un coureur automobile, où la différence entre se et $\varnothing$ constitue le seul signe que la rupture en (36) est mécanique tandis que celle en (37) est d'ordre psychologique:

(36) En fin de course quelque chose a cassé, je ne sais pas exactement quoi. C'est une alerte pour la suite du Championnat (AFP)

(37) "Il y a eu d'abord le premier incident dans la 3e spéciale, et le sentiment d'injustice quand Carlos Sainz a été aidé sans être sanctionné, explique-t-il. Et, lundi alors que j'attaquais, j'ai fait ce tonneau. Et là, quelque chose s'est cassé. Je me suis dit que je n'allais pas gagner. Je n'ai même plus eu envie de m'accrocher. Je me sentais détaché" (AFP)

\subsection{Rompre}

Les pourcentages dans le tableau 2 indiquent que rompre favorise la construction réfléchie de façon plus nette que casser, respectivement 68,1 / 36,7\% dans le corpus AFP et 58.3 / 86,2\% dans celui d'ARTFL. Cette différence s'accentue encore si l'on prend en compte la présence de l'expression fortement conventionalisées plier mais ne pas rompre illustrée en (38), qui, dans une forme ou une autre, figure dans 14 des 57 exemples du corpus AFP.

(38) Et, de fait, on a l'impression que cet homme plie mais ne rompt pas (AFP)

Dans les autres cas, les conditions qui régissent la sélection des deux constructions sont similaires à celles de casser. La construction simple tend à être choisie quand la participation du sujet de l'intransitif à la 
configuration dynamique qui cause sa rupture est défocalisée. Les exemples en (39) et (40) indiquent qu'ici aussi, cette défocalisation s'opère quand la rupture n'est pas directement pertinente au but narratif de la phrase:

(39) Pas de répit sur le front des inondations.... Trois barrages sous haute surveillance en Ille-etVilaine; une digue qui menace de rompre dans le Morbihan (AFP)

(40) On s'attendait à ce que les crues atteignent leur maximum mercredi après-midi. Les digues contenant les eaux de la Meuse et du Waal tenaient, mais la menace d'une rupture rendait les opérations d'évacuation urgentes. Si l'une des digues venait à rompre, les eaux pourraient submerger des routes secondaires et empêcher certains habitants de partir (AFP)

Dans (39) et (40), les passages décrivent les dangers d'inondations qui menacent une région. Le bris des digues représente l'un de ces dangers. L'accent est mis sur les conséquences des inondations pour la région, plutôt que sur l'aspect dynamique de la lutte de la digue contre la montée des eaux. Ces exemples peuvent être comparés à ceux en (41) et (42) où l'accent narratif est placé sur les digues elles mêmes, c'est à dire leur intégrité structurale et leur hypothétique résistance à la poussée de l'eau. La sélection de la construction réfléchie reflète l'intérêt de l'auteur pour leur capacité à endiguer cette poussée.

(41) A Ochten, en plein centre du pays, une digue, au pied de laquelle vivent 4.500 personnes, présentait des fissures inquiétantes et menaçait de se rompre...(AFP)

(42) La facture la plus salée sera celle de la rénovation des centaines de kilomètres de digues, construites au Moyen Age, qui ont menacé de se rompre sous la pression de l'eau. (AFP)

Enfin, ainsi que nous l'avions observé pour casser, la présentation de la rupture du sujet de l'intransitif comme faisant partie de façon stable et prévisible du monde tel qu'on le connaît favorise la présence de la forme simple Ø, comme le montre l'exemple en (43):

(43) Il faut se garder de couper ces liens avec la nouvelle lune, s' ils sont de chêne, et s' ils sont de noisetier, de saule, de bois blanc, avec la lune faite, ancienne. Ils ne se laissent point tordre, ils rompent infailliblement. (Pesquidoux J. de. Le Livre de Raison T. 1)

Les données présentées dans cette section montrent que pour casser et rompre, le choix de se et $\varnothing$ dépend de la volonté de l'auteur de mettre en valeur ou d'occulter le rôle dynamique du sujet de l'intransitif dans la configuration dynamique évoquée par le verbe. La différence de fréquence des deux constructions avec les deux verbes peut également être brièvement évoquée. Il semble en effet qu'elle soit partiellement imputable à certaines différences sémantiques subtiles entre casser et rompre. Il a déjà été signalé que rompre est fréquemment attesté avec des entités qui décrivent des situations d'équilibre (silence, harmonie, etc.), c'est à dire des entités à structure interne complexe où chaque élément contribue de façon identique à la stabilité de l'ensemble. Dans un emploi intransitif, ces structures complexes sont particulièrement compatibles avec la construction réfléchie qui met en valeur la dynamique interne qui produit leur stabilité. ${ }^{4}$

\section{Conclusion}

Les résultats obtenus dans cette présentation montrent que se et $\varnothing$ représentent deux configurations dynamiques différentes. Se décrit l'intéraction entre deux forces qui s'opposent, alors que $\varnothing$ ne reconnaît qu'une seule source d'énergie. La construction réfléchie met donc envaleur le coté dynamique de l'entité représentée par le sujet de l'intransitif, tandis que la construction simple le défocalise. Le sens des deux constructions se combine avec la structure sémantique spécifique des prédicats, ainsi que des considérations plus générales de stratégie discursive, pour rendre compte de leur distribution avec exploser, éclater, briser, casser, et rompre. Selon cette analyse, la distribution de se et $\varnothing$ répond à des 
motivations sémantiques similaires, quoique moins systématiques, à celles que Maldonado (1988) décrit pour l'espagnol.

L'analyse développée ici a des conséquences importantes pour un traitement global des prédicats de changement d'état. Remarquons en premier lieu qu'elle ne présente pas une rupture complète avec la tradition sémantique brièvement évoquée en introduction. Elle la suit tout d'abord en imputant la distribution des constructions simples et réfléchies à des configurations dynamiques spécifiques. De plus, elle traite également se comme une construction essentiellement énergique et $\varnothing$ comme une construction plus statique. Elle permet cependant de rendre compte des données relatives aux verbes de rupture de façon plus précise. Tout d'abord, il semble difficile d'évoquer une virtualité potentielle pour expliquer la présence de la construction simple avec éclater en (14)-(17), où des entités comme guerre, ou fusillade n'existent que par la dynamique des forces qui les créent. L'intégration des facteurs discursifs et sémantiques permet également une explication plus convaincante des cas d'alternation possible. Par example, en (1a) répété ici, la présence du participe en s'accrochant indique clairement que la rupture de la branche est occasionnée par des facteurs extérieurs. L'analyse de la présence de $\varnothing$ en termes de la potentialité virtuelle de la branche à casser ne semble donc pas particulièrement pertinente. En revanche, une explication en termes de la défocalisation de l'aspect dynamique de la branche dans l'épisode de rupture est moins problématique car intimement lié à des considérations discursives plus générales.

(1) a. Le jeune homme est monté sur le rebord d'une fenêtre au troisième étage de l'établissement et a voulu descendre en s'accrochant à la branche d'un arbre, mais elle a cassé, indique le journal The Statesman (AFP)

Si l'hypothèse présentée ici permet une analyse plus précise des verbes de rupture, il reste à déterminer si elle permet également de rendre compte de la forme de la construction intransitive avec l'ensemble des verbes de changement d'état. Une analyse préliminaire montre que c'est en effet le cas pour l'alternation durcir / se durcir, ainsi que pour la paire tomber / s'écrouler (Jones 1996), mais il reste beaucoup de travail à effectuer avant de pouvoir le prétendre. Quoi qu'il en soit, la présente étude montre la nécessité d'un travail systématique spécifique pour évaluer précisément le sens des constructions simples et réfléchies dans les sous classes sémantiques diverses qui constituent la catégorie générale.des verbes de changement d'état.

\section{Références}

Bailly, Charles. 1932. Linguistique Générale et Linguistique Française. Bern: Francke.

Dubois, Jean. 1964 Distribution, ensemble et marque dans le lexique. Cahiers de Lexicologie IV 1 : 5-16

Forest, Robert. 1988. Sémantisme entéléchique et affinité descriptive : Pour une réanalyse des verbes symétriques et neutres du Français. Bulletin de la Société Linguistique de Paris 83 : 137-162.

Goldberg, Adele. 1995. Constructions: A Construction Grammar Approach to Argument Structure. Chicago and London: University of Chicago Press.

Junker, Marie-Odile. 1987. Transitive, intransitive, and reflexive uses of deadjectival verbs in French. In Birdsong, David, and Jean Pierre Montreuil (eds.) Advances in Romance Linguistics. Dordrecht: Foris, 189-199.

Jones, Michael Allan. 1996. Foundations of French Syntax. Cambridge: Cambridge University Press.

Labelle, Marie. 1992. Change of State and valency. Journal of Linguistics 28: 375-414.

Lagane, René. 1967. Les verbes symétriques: Economie morpho-syntaxique et différentiation sémantique. Cahiers de Lexicologie X 1 : 21-30

Langacker, Ronald. W. 1987. Foundations of Cognitive Grammar (Vol. 1). Theoretical Prerequisites. Stanford: Stanford University Press.

Langacker, Ronald W. 1991. Foundations of Cognitive Grammar. Vol. 2: Descriptive Application. Stanford: Stanford University Press. 
DOI $10.1051 / \mathrm{cmlf0} 0844$

Maldonado, Ricardo. 1988. Energetic reflexives in Spanish. BLS 14: 153-165.

Rothemberg, Mira. 1974. Les Verbes à la Fois Transitifs et Intransitifs en Français Contemporain. Paris : Mouton.

Talmy, Leonard, 1988. Force dynamics in language and cognition. Cognitive Science 2: 49-100.

Trésor de la Langue Française. (1971). Paris. CNRS \& Gallimard.

1 Le choix de casser, briser, et rompre est motivé par leur proximité sémantique. Par exemple, Dubois (1964: 6) les traite comme les trois composantes d'un 'champ lexical'.

2 Les 5 exemples de la construction Ø avec briser proviennent du domaine maritime documenté dans le Trésor de la Langue Française (IV: 277)

3 Bien que l'unité soit en général considérée comme un état désirable, elle est parfois vue comme une contrainte, comme dans l'exemple (i), ce qui explique sa présence dans la table 3 avec briser.

(i) Car, sous le couvert de la psychologie, l'objet de Gide est secrètement métaphysique: il s'agit pour lui de briser l'unité que l'intelligence et la volonté introduisent dans la complexité sensible, d'en établir l'inanité en montrant dans leur intervention un principe de discorde et de trouble, car il tend à croire que l'unité, c'est- à- dire la béatitude, ne peut être atteinte que par l'abandon à notre nature animale. (Massi. H. Jugements T. 2

4 La présence plus fréquente de la construction simple dans le corpus AFP avec casser et rompre s'explique par l'importance du vocabulaire sportif qui comprend un grand nombre d'idiomes de la forme plier mais ne pas rompre, ainsi que de nombreux exemples du sens de casser 'voir ses progrès brutalement stoppés' illustré en (35). 